\title{
Associação entre sobrepeso, obesidade e transtornos mentais comuns em nutricionistas
}

\author{
Association between overweight, obesity and \\ common mental disorders in dietitians \\ Tânia Muzy da Silva', Odaleia Barbosa de Aguiar², Maria de Jesus Mendes da Fonseca
}

\section{RESUMO}

Objetivo: Analisar a associação entre sobrepeso, obesidade e transtornos mentais comuns em profissionais nutricionistas da rede pública de hospitais do município do Rio de Janeiro. Métodos: Estudo seccional, com 289 nutricionistas de hospitais públicos do município do Rio de Janeiro, de outubro de 2011 a agosto de 2012. Foi utilizado o índice de massa corporal $\left(\mathrm{kg} / \mathrm{m}^{2}\right)$ pela aferição de peso e altura, e os transtornos mentais comuns (TMC) pelo General Health Questionnaire (GHQ-12). Variáveis sociodemográficas (SES), laborativas e de saúde também foram incluídas no estudo. Resultados: As prevalências de sobrepeso, de obesidade e de TMC foram de 32,3\%, 15,3\% e 37,7\%, respectivamente. A análise múltipla não apresentou associação significativa após o ajuste pelas variáveis SES, laborativas e de saúde $(\mathrm{OR}=$ 0,60; IC95\% 0,32-1,10 para sobrepeso e $\mathrm{OR}=1,09$; IC95\% 0,50-2,37 para obesidade).

\section{Palavras-chave}

Saúde mental, sobrepeso, obesidade, nutricionista.

\section{Keywords}

Mental health, overweight, obesity, nutritionist.
Conclusão: Não encontramos associação entre sobrepeso, obesidade e TMC. Entretanto, as prevalências desses eventos na população estudada foram consideradas altas, o que aponta para a necessidade de estratégias de prevenção e promoção da saúde por se tratar de uma população de trabalhadoras envolvidas com o cuidado da população.

\section{ABSTRACT}

Objective: To analyze the association between overweight, obesity and common mental disorders in professional nutritionists from public hospitals in the city of Rio de Janeiro. Methods: Sectional study of 289 nutritionists in public hospitals in Rio de Janeiro, from October 2011 to August 2012. The body mass index $\left(\mathrm{kg} / \mathrm{m}^{2}\right)$ was used to measure height and weight, and common mental disorders (CMD) by the General Health Questionnaire (GHQ-12). Socio-demographic (socio-economic status - SES), labor-related and health variables were also included in the study. Results: The prevalence of overweight and obesity were $32.3 \%$ and $15.3 \%$ respectively and $37.7 \%$ of CMD. Multivariate analysis showed no significant association after adjusting for SES variables, work-related and health $(\mathrm{OR}=0.60,95 \% \mathrm{Cl} 0.32$ to 1.10 for overweight and $\mathrm{OR}=1.09,95 \% \mathrm{Cl} 0.50$ to 2.37 for obesity). Conclusion: There was no significant association between excess weight (overweight and obesity) and CMD. However were found high prevalence of overweight, obesity and CMD, which points to the need for health promotion and prevention strategies for dealing with a population of workers involved with the care of the population.

1 Universidade Federal do Rio de Janeiro (UFRJ), Instituto de Nutrição Josué de Castro.

2 Universidade do Estado do Rio de Janeiro (UERJ), Instituto de Nutrição, Departamento de Nutrição Aplicada.

3 Escola Nacional de Saúde Pública (Fiocruz), Departamento de Epidemiologia e Métodos Quantitativos em Saúde.

Endereço para correspondência: Tânia Muzy da Silva

Centro de Ciências da Saúde, Cidade Universitária

Av. Carlos Chagas Filho, 373, Bloco K, Sala K-14

DOI: 10.1590/0047-2085000000053 


\section{INTRODUÇÃO}

Excesso de peso e transtorno mental comum (TMC) são problemas de saúde pública importantes que vêm crescendo na população mundial e chamando a atenção dos pesquisadores para o estabelecimento de uma relação causal entre eles ${ }^{1,2}$. Além disso, esses agravos são fatores de risco para doenças crônicas e desempenham papel importante na morbimortalidade da população $0^{3,4}$.

A tendência secular do estado nutricional no Brasil avaliada pelos inquéritos populacionais (ENDEF, PNSN, POF) é de crescimento contínuo do excesso de peso e da obesidade ${ }^{5}$. Em 2008, as prevalências de excesso de peso e obesidade na população adulta se encontravam na ordem de $49 \%$ e $15 \%$, respectivamente, com aumento expressivo em todas as regiões do país. O comportamento, em relação a sexo e condições socioeconômicas, foi diferenciado, demonstrando que ambos exercem papel importante nesse contexto ${ }^{5}$. Esse quadro epidemiológico de excesso de peso está associado ao desequilíbrio entre ingestão e gasto de energia, sedentarismo e mudanças no padrão alimentar brasileiro ${ }^{5}$, com tendência crescente de substituição de alimentos tradicionais na dieta brasileira (como arroz, feijão e hortaliças) por bebidas e alimentos industrializados de alta densidade energética e baixo valor nutricional (como refrigerantes, biscoitos, carnes processadas e comida pronta) ${ }^{6}$.

Estimativas para prevalência de transtornos mentais comuns (TMC) abrangentes da população brasileira não foram encontradas, entretanto existem estudos de TMC em algumas cidades apontando para altas prevalências nessas populações ${ }^{7}$. Ludermir e Melo ${ }^{8}$ encontraram prevalência de 35\% de TMC em uma amostra de 621 adultos, na cidade de Olinda (PE). Em avaliação de 4.030 trabalhadores de uma universidade pública do Rio de Janeiro, Lopes et al. ${ }^{9}$ encontraram prevalências de TMC de 22\%. Fortes et al. ${ }^{10}$ analisaram pacientes atendidos em Programa de Saúde da Família no RJ, encontrando prevalência de 56\% de TMC. Um estudo multicêntrico realizado em unidades de saúde da família nas cidades de Rio de Janeiro, São Paulo, Porto Alegre e Fortaleza, em 2009 e 2010, demonstrou prevalências de 51,9\%, 53,3\%, 57,7\% e 64,3\%, apresentando associação significativa para as mulheres e os indivíduos de baixa renda $(p<0,05)^{11}$.

Os TMC são associados à baixa qualidade de saúde e de vida, à invalidez no trabalho e aos altos custos para a saúde pública e contribuem significativamente para a carga global de doenças no mundo. São mais prevalentes em mulheres, negros e populações em condições socioeconômicas menos privilegiadas, mas também em populações de trabalhadores, por causa do alto stress da vida moderna em decorrência da competitividade e globalização $0^{4,89}$.

Embora estejam bem estabelecidas as consequências que ambos os eventos, isoladamente, trazem para a saúde da população, a relação entre excesso de peso, em especial obesidade e transtornos mentais, especialmente a depressão, tem sido pouco estudada pelos pesquisadores e com resultados controversos. Porém, sabe-se que a forma como essa relação ocorre pode depender de alguns fatores como sexo, idade, grau de transtorno mental e de excesso de peso, assim como de outras condições sociodemográficas 1,2,2,13.

Tendo em vista a relevância desses agravos para a saúde pública, com crescimento alarmante de suas prevalências nas últimas décadas, assim como do prognóstico preocupante sobre a proporção desses agravos num cenário futuro de saúde da população, que são eventos com evidências de uma relação estabelecida, esta investigação tem como objetivo analisar a associação entre sobrepeso, obesidade e presença de transtornos mentais comuns em nutricionistas que trabalham em hospitais da rede pública municipal do Rio de Janeiro.

\section{MÉTODOS}

Estudo de desenho seccional, tipo censo, conduzido em todos os hospitais da rede pública do município do Rio de Janeiro, totalizando 23. Somente um hospital não participou, porque estava em processo de desativação.

\section{População do estudo}

Nutricionistas que tinham vínculo estatutário e prestadores de serviço das empresas fornecedoras de refeições de todas as unidades hospitalares foram convidados a participar do estudo. O total de nutricionistas lotados nos hospitais era de 305. Desse total, $15(4,9 \%)$ se recusaram a participar do estudo; uma demissão ocorreu durante a coleta de dados e não foi possível contatá-la (0,003\%), resultando em 289 nutricionistas avaliados.

\section{Coleta de dados e instrumentos}

O pré-teste do questionário multidimensional autoadministrável foi realizado com nutricionistas e alunos do curso de graduação de nutrição da UERJ, cujo objetivo era avaliar aspectos pertinentes à clareza de formulação das perguntas, possíveis resistências em responder a determinadas questões, adequação da sequência dos blocos temáticos e tempo necessário para o preenchimento. Um estudo piloto foi conduzido com os nutricionistas que trabalhavam no Instituto Annes Dias (órgão normativo e regulador das ações de alimentação e nutrição do município do Rio de Janeiro), com o objetivo de testar os procedimentos e materiais propostos para o trabalho de campo.

O treinamento da pesquisa foi constituído de duas etapas, com o auxílio do Manual do Entrevistador; a primeira para apresentação dos objetivos e leitura do manual e a segunda para padronização das técnicas de aferição das medidas antropométricas (peso e estatura). 
A coleta de dados foi realizada no período de outubro de 2011 a agosto de 2012, no próprio local de trabalho, com convite individual a cada nutricionista, no qual foi apresentado o questionário com a devida orientação para o preenchimento. A aferição das medidas antropométricas foi realizada no momento da visita do pesquisador.

O questionário continha seis blocos. O primeiro bloco considerava os dados do entrevistado (nome do hospital, data de preenchimento, data de nascimento, naturalidade e sexo); o segundo bloco, hábitos relacionados à alimentação e atividade física (compulsão alimentar, atividades físicas realizadas, informações sobre tabagismo e consumo de álcool); e o terceiro investigava a saúde e a qualidade de vida (autoavaliação do estado de saúde, escala GHQ-12, presença de doenças, capacidade de realizar atividades, sentimentos e experiências vividas considerando situações delicadas e de discriminação).

O quarto e o quinto bloco abordavam a história profissional e características do trabalho (escolaridade desde a graduação, tempo de formação e de trabalho, função de chefia, quantidade de locais de trabalho, tipo de vínculo, regime de trabalho, jornada de trabalho, situações do dia a dia de trabalho, contato com pacientes, ambiente de trabalho e ausências do trabalho) e a relação com a vizinhança onde reside. As condições socioeconômicas (renda familiar, situação conjugal, filhos, chefe de família, bens que possui e cor) foram tratadas no último bloco.

\section{Variável dependente, explicativa e covariáveis}

A variável dependente (estado nutricional) foi avaliada por meio do IMC, obtido pelo peso e altura aferidos (peso em $\mathrm{kg} /$ estatura em $\mathrm{m}^{2}$ ), considerando os pontos de corte de IMC preconizados pela Organização Mundial de Saúde14: baixo peso $\left(<18,5 \mathrm{~kg} / \mathrm{m}^{2}\right)$, eutrofia $\left(18,5-24,9 \mathrm{~kg} / \mathrm{m}^{2}\right)$, sobrepeso $\left(25-29,9 \mathrm{~kg} / \mathrm{m}^{2}\right)$ e obesidade (acima de $\left.30 \mathrm{~kg} / \mathrm{m}^{2}\right)$.

A variável explicativa (TMC) foi avaliada pela escala GHQ12, desenvolvida por Goldberg e Blackwell15, traduzida e validada para a população brasileira. Essa escala vem sendo utilizada para rastreamento de sinais e sintomas de transtornos mentais não psicóticos, ou seja, aqueles com menor gravidade e mais comumente encontrados na população ${ }^{16}$. Esse instrumento contém 12 itens, que cobrem sentimentos de tensão, depressão, incapacidade, medo de lidar com situações habituais, ansiedade e falta de confiança e investiga como o indivíduo tem passado nas últimas duas semanas. São quatro opções de resposta e ao final é feito o somatório e são considerados casos suspeitos de sinais e sintomas de transtornos mentais comuns, aqueles que apresentam escores $\geq 3$.

As covariáveis incluídas no estudo foram:

(i) condições sociodemográficas, tais como: idade em terços bivariada ( $\leq 37$ anos, $>37$ e $<47$ anos e $\geq 47$ anos), idade contínua na multivariada (anos), natura- lidade (Rio de Janeiro e outros estados), sexo (masculino e feminino), escolaridade (universitário, pós- graduação lato sensu e pós-graduação stricto sensu), renda familiar per capita em terços ( $\leq \mathrm{R} \$ 1.544,30$; $>R \$ 1.544,30 \mathrm{e}<\mathrm{R} \$ 2.271,00 \mathrm{e} \geq \mathrm{R} \$ 2.271,00$ ), situação conjugal (solteiro/separado/viúvo e casado), fiIhos (sim ou não) e cor da pele (negra, parda/outras e branca);

(ii) características laborativas: anos de trabalho como nutricionista (terços na univariada e contínua na multivariada); tipo de vínculo principal (CLT ou estatutário); regime de trabalho (diarista ou plantonista); outro vínculo de trabalho (sim ou não) e locais de trabalho na nutrição (um local e dois ou mais locais);

(iii) hábitos de vida e condições de saúde: prática de atividade física atribuída pela escala IPAQ, classificando os indivíduos como insuficientemente ativo e suficientemente ativo e morbidades (sim ou não) - hipercolesterolemia, diabetes, lesão por esforço repetitivo (LER) e hipertensão arterial sistêmica (HAS).

\section{Análise estatística}

$\mathrm{Na}$ etapa inicial foram calculadas as prevalências do estado nutricional e do TMC, bem como das covariáveis categóricas. As medidas de tendência central (média e mediana) e a medida de dispersão (desvio-padrão) foram calculadas para as covariáveis contínuas. Na análise bivariada, os testes de qui-quadrado ou de Fisher (caselas com $n<5$ ) foram utilizados para as variáveis categóricas e a ANOVA, para as variáveis contínuas, visando verificar a significância estatística, utilizando como critério de seleção para a análise multivariada valores de $p<0,20$, de forma a não excluir variáveis importantes. A análise multivariada foi realizada por regressão logística multinomial, considerando os dados completos dos participantes e excluindo os homens por não haver obesos entre eles, resultando num total de 266 indivíduos. O nível de significância considerado foi de $p<0,05$.

As medidas de associação foram expressas por meio da razão de chances (OR) e seus respectivos intervalos de confiança de 95\% (IC95\%). As variáveis socioeconômicas, de saúde e laborativas foram usadas para o ajuste e para possíveis efeitos de confundimentos.

Para avaliar a qualidade do ajuste do modelo final, foi utilizado o teste da razão de verossimilhança.

Os dados foram digitados em planilhas de Excel 2010, com digitação única e conferência por amostragem. As análises foram processadas no software R Studio versão $2.15^{17}$.

Este estudo teve aprovação do Comitê de Ética e Pesquisa da Secretaria Municipal de Saúde e Defesa Civil (CEPSMSDC-RJ), sob o protocolo no 0664.0.000.314-11. Todos os nutricionistas envolvidos no estudo assinaram um Termo de Consentimento Livre e Esclarecido. 


\section{RESULTADOS}

A média de idade da população estudada foi de 41 anos (DP = 10,0); a maioria era do sexo feminino, casada, com filhos e de cor branca; e 48,4\% tinham curso de pós-graduação lato sensu e stricto sensu (Tabela 1). Com relação aos aspectos laborais (Tabela 2), 64,5\% tinham menos de 21 anos de trabaIho como nutricionista, sendo o tempo médio de 17 anos $(\mathrm{DP}=9,9)$. Quanto ao tempo de trabalho na empresa e/ou município, a média era de 13 anos $(D P=9,5)$. A maioria era de funcionários públicos com regime de trabalho na forma de "plantão" e somente um vínculo de trabalho.

Tabela 1. Características sociodemográficas de nutricionistas de hospitais da rede municipal de saúde do Rio de Janeiro, RJ, Brasil 2011/2012

\begin{tabular}{|c|c|c|c|c|}
\hline \multirow{2}{*}{ Variáveis } & \multirow{2}{*}{$\mathrm{n}$} & \multicolumn{2}{|c|}{ Frequência } & \multirow{2}{*}{ IC95\% } \\
\hline & & $\mathrm{n}$ & $\%$ & \\
\hline Faixa etária & 289 & & & \\
\hline 20 a 30 anos & & 52 & 18,0 & $13,74-22,92$ \\
\hline 31 a 40 anos & & 81 & 28,0 & $22,9-33,59$ \\
\hline 41 a 50 anos & & 98 & 33,9 & $28,47-39,69$ \\
\hline 51 a 69 anos & & 58 & 20,1 & $15,60-25,16$ \\
\hline Sexo & 289 & & & \\
\hline Masculino & & 08 & 2,8 & $1,2-5,38$ \\
\hline Feminino & & 281 & 97,2 & $94,61-98,79$ \\
\hline Escolaridade & 289 & & & \\
\hline Universitário & & 149 & 51,6 & $45,62-57,45$ \\
\hline Pós-graduação lato sensu & & 127 & 43,9 & $38,13-49,87$ \\
\hline Pós-graduação stricto sensu & & 13 & 4,5 & $24,16-75,69$ \\
\hline Renda familiar per capita (terço) & 280 & & & \\
\hline$\leq R \$ 1.544,30$ & & 106 & 37,9 & $32,15-43,82$ \\
\hline$>\mathrm{R} \$ 1.544,30 \mathrm{e}<\mathrm{R} \$ 2.271,00$ & & 65 & 23,2 & $18,39-28,60$ \\
\hline$\geq R \$ 2.271,00$ & & 109 & 38,9 & $33,18-44,91$ \\
\hline Situação conjugal & 287 & & & \\
\hline Solteiro/viúvo/separado & & 137 & 47,7 & $41,83-53,68$ \\
\hline Casado & & 150 & 52,3 & $46,31-58,17$ \\
\hline Filhos & 286 & & & \\
\hline Sim & & 156 & 54,5 & $39,58-51,42$ \\
\hline Não & & 130 & 45,5 & $48,57-60,41$ \\
\hline Cor & 289 & & & \\
\hline Parda/negra/outras & & 84 & 29,1 & $23,89-34,67$ \\
\hline Branca & & 205 & 70,9 & $65,32-76,10$ \\
\hline
\end{tabular}

Quanto aos hábitos de vida e saúde (Tabela 2), a maioria era sedentária (72,5\%) e $26 \%$ apresentavam comorbidades, tais como: hipercolesterolemia (26\%), LER (20,5\%), hipertensão arterial $(20,1 \%)$ e diabetes (2,8\%) (dados não apresentados na tabela 2). A prevalência de TMC foi de $37,7 \%$ e a de excesso de peso foi de $47,6 \%$, sendo $15,3 \%$ para obesidade. O IMC médio foi de $25,7 \mathrm{~kg} / \mathrm{cm}(\mathrm{DP}=4,83)$.
Tabela 2. Características laborativas e de saúde dos nutricionistas de hospitais da rede municipal de saúde do Rio de Janeiro, RJ, Brasil 2011/2012

\begin{tabular}{|c|c|c|c|c|}
\hline \multirow{2}{*}{ Variáveis } & \multirow{2}{*}{$\mathrm{n}$} & \multicolumn{2}{|c|}{ Frequência } & \multirow{2}{*}{ IC95\% } \\
\hline & & $\mathrm{n}$ & $\%$ & \\
\hline Anos de trabalho (nutricionista) & 288 & & & \\
\hline$\leq 12,7$ anos & & 96 & 33,3 & $27,91-39,10$ \\
\hline$>12,7 \mathrm{e}<20,9$ anos & & 90 & 31,2 & $25,94-36,95$ \\
\hline$\geq 21$ anos & & 102 & 35,4 & $29,89-41,24$ \\
\hline Tipo de vínculo principal & 289 & & & \\
\hline Estatutário & & 188 & 65,1 & $59,24-70,54$ \\
\hline CLT & & 101 & 34,9 & $29,45-40,75$ \\
\hline Regime de trabalho & 288 & & & \\
\hline Diarista & & 68 & 23,6 & $18,82-28,94$ \\
\hline Plantonista & & 220 & 76,4 & $71,05-81,17$ \\
\hline Outro vínculo de trabalho & 289 & & & \\
\hline Sim & & 94 & 32,5 & $61,74-72,84$ \\
\hline Não & & 195 & 67,5 & $27,15-38,26$ \\
\hline Locais de trabalho na nutrição & 289 & & & \\
\hline 1 local & & 177 & 61,2 & $55,36-66,89$ \\
\hline $20 u+$ locais & & 112 & 38,8 & $33,1-44,63$ \\
\hline Atividade física & 284 & & & \\
\hline Insuficientemente ativo & & 206 & 72,5 & $66,58-77,31$ \\
\hline Suficientemente ativo & & 78 & 27,5 & $21,7-32,31$ \\
\hline Comorbidades* & 289 & & & \\
\hline Ausência & & 214 & 74,0 & $68,59-79,0$ \\
\hline Presença & & 75 & 26,0 & $20,1-31,1$ \\
\hline Transtornos mentais comuns & 284 & & & \\
\hline Ausência & & 177 & 62,3 & $56,61-68,20$ \\
\hline Presença & & 107 & 37,7 & $32,13-43,74$ \\
\hline Estado nutricional & 288 & & & \\
\hline Eutrofia & & 151 & 52,4 & $46,48-58,32$ \\
\hline Sobrepeso & & 93 & 32,3 & $11,32-19,96$ \\
\hline Obesidade & & 44 & 15,3 & $26,92-38,02$ \\
\hline
\end{tabular}

Observamos maiores prevalências de sobrepeso entre aqueles: que relataram comorbidades (40\%); que se encontravam na faixa etária mais elevada (> 47 anos $-41,6 \%$ ); com maior tempo de trabalho como nutricionista ( $>21$ anos 41,2\%); com mais de um vínculo empregatício $(39,4 \%)$ e que informaram ter cor negra ou parda (40,5\%). Com relação à obesidade, foram encontradas maiores prevalências entre aqueles que apresentavam TMC (17,8\%), não tinham outro vínculo empregatício (19,1\%), com vínculo de CLT $(21,0 \%)$ e que tinham menor renda (22,6\%) (Tabela 3).

Com base nos resultados do modelo múltiplo da tabela 4, pode-se observar que os transtornos mentais comuns não se apresentaram associados estatisticamente $(p<0,05)$ ao sobrepeso e à obesidade, porém destaca-se a magnitude da 
associação entre TMC e obesidade na análise bruta (OR 1,32; 0,64-2,72), que teve redução da magnitude no modelo final (OR 1,09; IC95\% 0,50-2,37) e para o sobrepeso houve diminuição da chance com intervalos limítrofes e estatisticamente significativos a $10 \%$ nos últimos modelos.

\section{DISCUSSÃO}

Ainda há muita controvérsia sobre o impacto da obesidade na saúde mental e vice-versa, não havendo clareza nessa direção de causalidade apontando para uma bidirecionalidade. Para elucidar essa controvérsia, alguns mecanismos têm sido propostos sobre a relação entre as doenças. Os obesos travam uma verdadeira batalha para a perda de peso, sofrem com baixa autoestima pelo estigma social a que estão sujeitos, especialmente as mulheres, como também são mais sedentários; por outro lado, o indivíduo com depressão faz uso de drogas psicotrópicas que promovem hiperfagia, são mais sedentários e tendem a comer mais para diminuir a ansiedade ${ }^{18}$.

No estabelecimento dessa relação, deve-se considerar que ambos os eventos são condições de saúde, resultados de um conjunto de fatores de risco ou de proteção que se acumulam ao longo da vida, tornando essa associação complexa e que deve levar em conta o contexto social e cultural dos indivíduos, o tempo de exposição, assim como os aspectos biológicos que podem mediar essa relação.

Tabela 3. Análise bivariada - variável desfecho, variável de exposição e covariáveis que apresentaram significância estatística na análise bivariada $(p<0,20)^{*}$

\begin{tabular}{|c|c|c|c|c|c|c|c|c|}
\hline \multirow{2}{*}{ Variáveis } & \multirow{2}{*}{$\mathrm{n}$} & \multicolumn{2}{|c|}{ Eutrofia } & \multicolumn{2}{|c|}{ Sobrepeso } & \multicolumn{2}{|c|}{ Obesidade } & \multirow{2}{*}{$p$ valor } \\
\hline & & $n$ & $\%$ & $n$ & $\%$ & $\mathrm{n}$ & $\%$ & \\
\hline Transtornos mentais comuns & 283 & & & & & & & 0,25 \\
\hline Ausência & & 90 & 51,1 & 63 & 35,8 & 23 & 13,1 & \\
\hline Presença & & 59 & 55,1 & 29 & 27,1 & 19 & 17,8 & \\
\hline Comorbidades & 288 & & & & & & & 0,13 \\
\hline Ausência & & 119 & 55,9 & 63 & 29,6 & 31 & 14,6 & \\
\hline Presença & & 32 & 42,7 & 30 & 40,0 & 13 & 17,3 & \\
\hline Idade & 288 & & & & & & & 0,11 \\
\hline$\leq 37$ anos & & 56 & 57,7 & 28 & 28,9 & 13 & 13,4 & \\
\hline 37,1 a 47 anos & & 52 & 57,8 & 23 & 25,6 & 15 & 16,7 & \\
\hline$\geq 47$ anos & & 43 & 42,6 & 42 & 41,6 & 16 & 15,8 & \\
\hline Anos de trabalho como nutricionista & 287 & & & & & & & 0,08 \\
\hline$\leq 12,7$ anos & & 56 & 58,9 & 28 & 29,5 & 11 & 11,6 & \\
\hline 12,8 a 20,9 anos & & 52 & 57,8 & 23 & 25,6 & 15 & 16,7 & \\
\hline$\geq 21$ anos & & 43 & 42,2 & 42 & 41,2 & 17 & 16,7 & \\
\hline Outro vínculo & 288 & & & & & & & 0,02 \\
\hline Não & & 101 & 52,1 & 56 & 28,9 & 37 & 19,1 & \\
\hline Sim & & 50 & 53,2 & 37 & 39,4 & 07 & 7,4 & \\
\hline Tipo de vínculo & 288 & & & & & & & 0,14 \\
\hline Estatutário & & 102 & 54,3 & 63 & 33,5 & 23 & 12,2 & \\
\hline CLT & & 49 & 49,0 & 30 & 30,0 & 21 & 21,0 & \\
\hline Renda per capita (terço) & 279 & & & & & & & 0,008 \\
\hline Até $R \$ 1.544,30$ & & 45 & 42,5 & 37 & 34,9 & 24 & 22,6 & \\
\hline $\mathrm{R} \$ 1.544,31$ a $\mathrm{R} \$ 2.271,00$ & & 34 & 52,3 & 22 & 33,8 & 09 & 13,8 & \\
\hline Acima de $R \$ 2.271,00$ & & 67 & 62,0 & 34 & 31,5 & 07 & 6,5 & \\
\hline Cor & 288 & & & & & & & 0,14 \\
\hline Negra/Parda & & 40 & 47,6 & 34 & 40,5 & 10 & 11,9 & \\
\hline Branca & & 111 & 54,4 & 59 & 28,9 & 34 & 16,7 & \\
\hline
\end{tabular}

* Bivariada: teste $\chi^{2}$ e teste exato de Fisher $(n<5)$ e ANOVA para variáveis contínuas. 
Tabela 4. Análise múltipla - modelos de regressão logística multinomial para estado nutricional (nutricionistas de hospitais da rede municipal de saúde do Rio de Janeiro, RJ, Brasil, 2011/2012)

\begin{tabular}{|c|c|c|c|}
\hline Modelos $n=266$ & \multicolumn{2}{|c|}{$\begin{array}{c}\text { TMC } \\
\text { OR ajustado (IC95\%) }\end{array}$} & $p$ valor \\
\hline Sobrepeso & 0,67 & $0,38-1,18$ & 0,17 \\
\hline Obesidade & 1,32 & $0,64-2,72$ & 0,44 \\
\hline \multicolumn{4}{|l|}{ Modelo $2^{*}$} \\
\hline Sobrepeso & 0.67 & $0,37-1,20$ & \\
\hline Obesidade & 1.33 & $0,64-2,74$ & 0,43 \\
\hline \multicolumn{4}{|l|}{ Modelo $3^{*}$} \\
\hline Sobrepeso & 0,68 & $0,38-1,25$ & 0,19 \\
\hline Obesidade & 1,25 & $0,60-2,60$ & 0,54 \\
\hline \multicolumn{4}{|l|}{ Modelo $4^{*}$} \\
\hline Sobrepeso & 0,67 & $0,37-1,20$ & 0,17 \\
\hline Obesidade & 1,30 & $0,62-2,72$ & 0,48 \\
\hline \multicolumn{4}{|l|}{ Modelo $5^{*}$} \\
\hline Sobrepeso & 0,64 & $0,36-1,17$ & 0,14 \\
\hline Obesidade & 1,19 & $0,56-2,53$ & 0,65 \\
\hline \multicolumn{4}{|l|}{ Modelo $6^{*}$} \\
\hline Sobrepeso & 0,60 & $0,33-1,09$ & 0,09 \\
\hline Obesidade & 1,08 & $0,49-2,35$ & 0,84 \\
\hline \multicolumn{4}{|l|}{ Modelo $7^{*}$} \\
\hline Sobrepeso & 0,60 & $0,32-1,10$ & 0,09 \\
\hline Obesidade & 1,09 & $0,50-2,37$ & 0,83 \\
\hline
\end{tabular}

Categoria de referência: eutrofia.

* Modelo 1: Estado nutricional + TMC (binária) + morbidades (binária).

** Modelo 2: Estado nutricional + TMC (binária) + morbidades (binária) + idade (contínua)

*** Modelo 3: Estado nutricional + TMC (binária) + morbidades (binária) + idade (contínua) + anos de trabalho como nutricionista (contínua).

**** Modelo 4: Estado nutricional + TMC (binária) + morbidades (binária) + idade (contínua) + anos de trabalho como nutricionista (contínua) + outro vínculo profissional (binária).

***** Modelo 5: Estado nutricional + TMC (binária) + morbidades (binária) + idade (contínua) + anos de trabalho como nutricionista (contínua) + outro vínculo profissional (binária) + tipo do vínculo principal (CLT ou estatutária).

****** Modelo 6: Estado nutricional + TMC (binária) + morbidades (binária) + idade (contínua) + anos de trabalho como nutricionista (contínua) + outro vínculo profissional (binária) + tipo do vínculo principal (CLT ou estatutária) + terço de renda per capita (3 categorias).

******* Modelo 7: Estado nutricional + TMC (binária) + morbidades (binária) + idade (contínua) + anos de trabalho como nutricionista (contínua) + outro vínculo profissional (binária) + tipo do vínculo principal (CLT ou estatutária) + terço de renda per capita ( 3 categorias) + cor. $p<0,05$

Em nosso estudo, os resultados não demonstraram associação significativa entre sobrepeso e obesidade e transtornos mentais comuns. Esses achados são consistentes com alguns estudos realizados em populações ${ }^{19-21}$ e contrários a outros estudos que encontraram associação entre transtornos mentais e excesso de peso, especialmente obesidade e depressão $0^{22,23}$.

Atlantis e Baker', em revisão sistemática de estudos de coorte e seccionais, encontraram resultados nos estudos de coorte suportando a associação de obesidade e depressão que não foi encontrada na maioria dos estudos seccionais. McElroy et al. ${ }^{24}$ analisaram estudos clínicos e comunitários sobre mudanças de humor e sobrepeso e obesidade, destacando que, apesar de essa relação às vezes aparecer por coincidência, os resultados apontam que pode haver sim uma relação entre esses eventos. Outra revisão sistemática de estudos sobre obesidade e comorbidade psicológica também encontrou resultados conflitantes e com uma relação explicativa ainda a ser esclarecida ${ }^{25}$. Wit et al. ${ }^{2}$ realizaram metanálise de estudos comunitários sobre essa relação, evidenciando forte associação para as mulheres, mas não encontrando evidência para nenhum outro fator de confundimento.

Com base em dados de uma coorte de 4.154 funcionários públicos britânicos, Kivimaki et al.22 analisaram a associação entre obesidade e transtornos mentais comuns em cada fase do estudo, encontrando evidência dessa associação na última fase comparada tanto para homens $(1,31$; IC95\% 1,08$1,60)$ quanto para mulheres $(1,30$; IC95\% 1,02-1,67), quando ajustados para idade, etnia, estado civil, posição socioeconômica e uso de drogas psicotrópicas.

Uma investigação seccional utilizando dados de uma pesquisa de saúde com 15.099 mulheres espanholas encontrou associação estatisticamente significativa entre sofrimento psíquico, sobrepeso $(1,17$; IC95\% 1,01-1,38) e obesidade $(1,26$; IC95\% 1,02-1,55). Esse resultado foi obtido depois de controladas as variáveis: idade, doenças crônicas concomitantes (HAS, doença coronariana ou infarto no miocárdio), diabetes mellitus e atividade física, utilizadas na análise, demonstrando que essa relação existe e precisa ser mais estudada ${ }^{23}$.

No Brasil, encontramos resultados semelhantes aos nossos, em estudo realizado com funcionários de uma universidade pública do Rio de Janeiro ${ }^{26}$ para analisar a associação entre IMC, percepção corporal e transtornos mentais comuns, no qual se observou tendência de associação ( $p$ da tendência $=0,05$ ) entre IMC e TMC para mulheres, mas sem significância estatística quando ajustado por idade, renda, atividade física recreativa e problemas de saúde autorreferidos (OR 1,26, IC95\% 0,93-1,72). A semelhança com os nossos resultados pode ser pela similaridade da população de trabaIhadores que prestam serviços para a administração pública e pelo fato de que talvez outros fatores, como condições de trabalho e renda, possam ter influência maior no desenvolvimento do TMC e da obesidade.

Nosso estudo encontrou uma associação negativa para o sobrepeso (OR 0,60, IC95\% 0,32-1,10), também demonstrada no estudo de Veggi et al. ${ }^{26}$ para as mulheres (OR 0,97, IC95\% 0,75-1,25).

Um aspecto importante a ser destacado em nosso estudo quando comparamos os modelos da análise múltipla é a observação de que, com o acréscimo das variáveis tipo de vínculo profissional e renda per capita em terço, houve diminuição mais acentuada na magnitude, demonstrando que tais variáveis podem ter maior influência sobre essa relação. 
Esse achado também foi demonstrado no estudo de Veggi et al. ${ }^{26}$ para as mulheres, quando incluída a renda, mas não por Kivimaki et al. ${ }^{22}$, quando ajustado pelas variáveis socioeconômicas. Essas discrepâncias podem ser devidas a diferenças culturais e na estrutura de trabalho e renda entre os trabaIhadores do Brasil e os da Inglaterra.

Esta investigação apresenta como limitações: o desenho de estudo seccional, por não permitir conclusões de temporalidade e natureza causal; a homogeneidade da população estudada, que dificultou analisar as diferenças sobre fatores adjacentes tais como sexo, pela ausência de representação do sexo masculino na população; assim como a escolaridade, já que todos os profissionais tinham no mínimo nível superior, considerado de alta escolaridade (> 12 anos), não permitindo a comparação entre os níveis de escolaridade. Essa pode ser uma das razões para não termos encontrado associação significativa entre os eventos, considerando que o TMC é mais prevalente entre indivíduos com menor escolaridade ${ }^{8}$. Entretanto, a idade média acima de 40 anos favoreceria o aparecimento dessa relação, pois tanto a obesidade quanto o TMC aumentam com a idade ${ }^{5,7}$. Uma explicação seria a especificidade da população estudada: ativa, inserida em uma unidade de saúde, de nível socioeconômico mais alto que a população geral - características importantes de proteção para o desenvolvimento dos eventos de interesse.

Como pontos fortes, destacamos: a utilização de medidas antropométricas aferidas e não autorrelatadas; a utilização da escala GHQ, amplamente utilizada em estudos ocupacionais; o fato de esta ser a primeira investigação sobre saúde física e mental de nutricionistas, o que servirá de estímulo para outros estudos.

Apontamos como perspectiva futura para melhor avaliação da relação entre IMC e sofrimento psíquico, considerando que este possui caráter intermitente e se desenvolve em episódios, a necessidade da realização de estudos prospectivos com ondas mais curtas de seguimento, que possibilitarão identificar qual o papel dos agentes etiológicos com efeito recíproco e as diferenças em relação às variáveis sociodemográficas, bem como de estudos clínicos que permitam identificar mecanismos biológicos que possam influenciar nessa relação. É importante ressaltar o uso de instrumentos padronizados na avaliação de transtornos psiquiátricos, permitindo melhor comparabilidade dos resultados.

\section{CONCLUSÃO}

Não observamos associação significativa entre excesso de peso (sobrepeso e obesidade) e TMC. Entretanto, foram encontradas altas prevalências dos eventos analisados, o que aponta para a necessidade de estratégias de prevenção e promoção da saúde, por se tratar de uma população de trabalhadoras envolvidas com o cuidado da população.

\section{CONTRIBUIÇÕES INDIVIDUAIS}

Tânia Muzy da Silva - Participou da coleta, análise e interpretação dos dados e concepção do artigo.

Odaleia Barbosa de Aguiar - Participou da elaboração do desenho da pesquisa, coleta, análise e interpretação dos dados e concepção do artigo.

Maria de Jesus Mendes da Fonseca - Participou da elaboração do desenho da pesquisa, coleta, análise e interpretação dos dados e concepção do artigo.

\section{CONFLITOS DE INTERESSE}

Este estudo foi apoiado pelo Conselho Nacional de Desenvolvimento Científico e Tecnológico (CNPq), processo 402430/2010-7. Não existem conflitos de interesse.

\section{REFERÊNCIAS}

1. Atlantis $E$, Baker M. Obesity effects on depression: systematic review of epidemiological studies. Int J Obes (Lond). 2008;32(6):881-91.

2. Wit L, Luppino F, Straten AV, Penninx B, Zitman F, Cuijpers P. Depression and obesity: a meta-analysis of community-based studies. Psychiatry Res. 2010;178(2):230-5.

3. Organização Pan-Dyspepsia in diabetes type 1 da Saúde. Doenças crônico-degenerativas e obesidade: estratégia mundial sobre alimentação saudável, atividade física e saúde. 2003. 60p. Disponível em: <http://who.int/hpr/gs.facts.shtml>. Acesso em: 16 out, 2012.

4. Organização Mundial da Saúde. Relatório sobre a saúde no mundo: saúde mental nova concepção, Esperança Nova. Geneva. 2001. Disponível em: <http://www.who.int/ whr/2001/es/index.html >. Acesso em: 16 out, 2012.

5. Instituto Brasileiro de Geografia e Estatística. Pesquisa de Orçamentos Familiares 20082009: análise da disponibilidade domiciliar de alimentos e do estado nutricional no Brasil. Rio de Janeiro: IBGE. 2010. 88p. Disponível em: <http://www.ibge.gov.br/home/xml/ pof_2008_2009.shtm>. Acesso em: 19 out, 2012.

6. Levy-Costa RB, Sichieri R, Pontes NS, Monteiro CA. Disponibilidade domiciliar de alimentos no Brasil: distribuição e evolução (1974-2003). Rev Saúde Pública. 2005;39(4):530-40.

7. Fonseca MLG, Guimarães MBL, Vasconcelos EM. Sofrimento difuso e transtornos mentais comuns: uma revisão bibliográfica. Rev APS. 2008;11(3):285-94.

8. Ludermir AB, Melo Filho DA. Living conditions and occupational organization associated with common mental disorders [in Portuguese]. Rev Saúde Pública. 2002;36(2):213-21.

9. Lopes CS, Faerstein E, Chor D. Eventos de vida produtores de estresse e transtornos mentais comuns: resultados do Estudo Pró-Saúde. Cad Saude Publica. 2003;19(6):1713-20.

10. Fortes F, Villano LAB, Lopes (S. Perfil nosológico e prevalência de transtornos mentais comuns em pacientes atendidos em unidades do Programa de Saúde da Família (PSF) em Petrópolis, Rio de Janeiro. Rev Bras Psiquiatr. 2008:30(1):32-7.

11. Gonçalves DA, Mari JJ, Bower P, Gask L, Doerick C, Tófoli LF, et al. Brazilian multicentre study of common mental disorders in primary care: rates and related social and demographic factors. Cad Saude Publica. 2014;30(3):623-32.

12. Angela GFB, Ngo LH, Wee CC. The relationship between symptoms of depression and body weight in younger adults. Obesity. 2012;20(9):1922-8.

13. Simon GE, Korff MV, Saunders K, Miglioretti DL, Crane PK, Belle GV, et al. Association between obesity and psychiatric disorders in the us adult population. Arch Gen Psychiatry. 2006;63(7):824-30.

14. WHO. Obesity: preventing and managing the global epidemic. Report of WHO Consultation on Obesity. World Health Organization. Geneva, 1998. Disponível em: <http://www. who.int/nutrition/publications/obesity/WH0_TRS_894/en/>. Acesso em: 8 dez, 2012.

15. Goldberg DP, Blackwell B. Psychiatric illness in general practice. A detailed study using a new method of case identification. Br Med J. 1970;2(5707):439-43. 
16. Mari JJ, Williams P. A comparison of the validity of two psychiatric screening questionnaires (GHQ-12 and SRQ-20) in Brazil, using relative operating characteristic (ROC) analysis. Psychol Med. 1985;15(3):651-9.

17. R Development Core Team [computer program]. A language and environment for statistical computing. Foundation for Statistical Computing, Vienna, Austria. Disponível em: <http:// www.R-project.org/>. Acesso em: 10 jun, 2012.

18. Ross CE. Overweight and depression. J Health Soc Behav. 1994;35(1):63-78.

19. Bruffaerts R, Demyttenaere K, Vilagut G, Martinez M, Bonnewyn A, Graaf RD, et al. The relation between body mass index, mental health and functional disability: A European population perspective. Can J Psychiatry. 2008;53(10):679-88.

20. Kim JY, Chang HM, Cho JJ, Yoo SH, Kim SY. Relationship between obesity and depression in the Korean working population. J Korean Med. 2010;25(11):1560-7.

21. Sunwoo YK, Bae JN, Hahm BJ, Lee DW, Park JI, Cho SJ, et al. Relationships of mental disorders and weight status in the Korean adult population. J Korean Med. 2011;26(1):108-15.
22. Kivimaki M, Batty GD, Singh-Manoux A, Nabi H, Sabia S, Tabak AG, et al. Association between common mental disorder and obesity over the adult life course. Br J Psychiatr. 2009;195(2):149-55.

23. Martín-López R, Pérez-Farinós N, Hernández-Barrera V, Andres AL, Carrasco-Garrido P, Jiménez-García R. The association between excess weight and self-rated health and psychological distress in women in Spain. Public Health Nutr. 2011;14(7):1259-65.

24. McElroy SL, Kotwal L, Malhotra S, Nelson EB, Keck PE, Nemeroff (B. are mood disorders and obesity related? A review on the mental health professional. J Clin Psychiatry. 2004;65(5):634-51.

25. Baile Jl, Gonzalez MJ. Comorbilidad psicopatológica en obesidad. Anales Sis San Navarra. 2011;34(2):253-61.

26. Veggi $A B$, Lopes $C S$, Faerstein E, Sichieri R. Índice de massa corporal, percepção do peso corporal e transtornos mentais comuns entre funcionários de uma universidade no Rio de Janeiro. Rev Bras Psiquiatr. 2004;26(4):242-7. 